\title{
WoMAN WITH AIDS AND STATUS EPILEPTICUS
}

\author{
Bonnie Callahan, $M D$
}

\section{Case Presentation}

A 42 year-old female with a medical history of HIV presented to the Emergency Department with nausea and vomiting for the past five days. Six days prior to presentation, she had one week of symptoms of progressive dyspnea and non-productive cough which were evaluated by her primary care physician. At that time, blood work revealed a CD 4 count of 150 cells $/ \mathrm{mm} 3$ and a chest $\mathrm{x}$-ray was suggestive of pneumonia. She was given a prescription of trimethoprim-sulfamethoxazole (TMP/SMX) and guaifenesin with codeine. Within twenty-four hours of starting the prescribed medications, she developed nausea and vomiting, and she stopped taking both medications three days prior to presentation. Although her cough and shortness-of-breath were much improved, her nausea and vomiting had not improved with discontinuation of the TMP/SMX and guaifenesin with codeine. She reported she was unable to tolerate any solid foods and was only tolerating small amounts of liquids. She also noted some associated epigastric "crampiness." Review of systems was notable for a new headache that started the morning of presentation to the hospital. The headache was bilateral, frontal, and "achy, non-throbbing." She had no associated photophobia, vision change, or neck-stiffness. She reported no fever, chills, chest pain, hemoptysis, diarrhea, or constipation. She had no other complaints.

On review of the patient's past medical history, she was noted to have type 2 diabetes, hypertension, "mild" asthma (never requiring hospitalization or intubation) and HIV/AIDS with a recent CD 4 count of 150 cells/mm3. Her surgical history included a TAH-BSO and breast biopsy. She was allergic to penicillin, which caused anaphylaxis. Her medications included glipizide, amlodipine, losartan, hydrochlorothiazide, and albuterol. Social history was negative for tobacco, alcohol, or substance abuse. The patient worked in an office setting and lived at home with her daughter. She reported no recent sick contacts and no recent travel. Her family history was significant for type 2 diabetes in her mother, who died from a stroke at age sixty, and breast cancer in her sister, who died from a stroke in her fifties.

In the ED, the patient was afebrile and her vital signs included a heart rate of 92 beats per minute, respiratory rate of 16 breaths per minute, blood pressure of $128 / 94 \mathrm{mmHg}$, and an oxygen saturation of $98 \%$ on room air. She was alert and oriented and in no acute distress. Physical exam revealed oral thrush and scattered expiratory wheezes in bilateral lungs with decreased breath sounds at bilateral bases. Her abdomen was diffusely tender, with normoactive bowel sounds throughout and no rebound or guarding. No masses or organomegaly were appreciated. The physical exam was otherwise unremarkable.

The initial evaluation included a chest x-ray which showed "hilar lymphadenopathy with upper lobe interstitial lung disease" which was thought to be secondary to infectious etiology. Her urinalysis was negative for infection, and a complete blood count showed a normal white blood cell count, a normal platelet count, and mild anemia with hemoglobin of $12 \mathrm{~g} / \mathrm{dL}$. She had an elevated lactate dehydrogenase of 287 IU/L. Her blood chemistries were normal, with the exception of a creatinine of $2.0 \mathrm{mg} / \mathrm{dL}$, with a known baseline of $0.8 \mathrm{mg} / \mathrm{dL}$ one year earlier. Blood and sputum cultures were collected. The patient was diagnosed with communityacquired pneumonia and was started on moxifloxacin in the ED. She was also started on intravenous TMP/SMX for possible PCP and fluconazole for her oral thrush. She was admitted to the hospital and started on intravenous fluid resuscitation.

The first night of the patient's hospital stay was uneventful. The following morning she was noted to have acute mental status change. She appeared awake, but would not answer questions or follow commands. She had a low grade temperature of $100^{\circ} \mathrm{F}$, was tachycardic at 110 beats per minute, with a blood pressure of $132 / 62 \mathrm{mmHg}$, a respiratory rate of 20 breaths per minute, and an oxygen saturation of $99 \%$ on room air. Her physical exam was unchanged from the previous day with the exception that she could not cooperate for the neurologic exam. Her pupils were equal and reactive to light. She did grimace in reaction to painful stimuli. Her muscle tone was noted to be normal with no rigidity. She was spontaneously moving all four extremities, but not on command. The patient was transferred to the telemetry floor and a full neurologic evaluation was initiated. A lumbar puncture was planned, cultures were obtained, and a head CT was unremarkable, showing no intracranial hemorrhage, acute territorial infarct, or cerebral edema. Additionally, no mass effect or midline shift was seen on the CT scan. The patient's fever increased to $103^{\circ} \mathrm{F}$, and the infectious disease specialist saw her in consult.

The patient's condition remained unchanged until 4:45 am the next morning, when a nurse witnessed the patient having tonicclonic seizure activity. During the seizure the patient foamed at the mouth and desaturated to $81 \%$ on $2 \mathrm{~L}$ oxygen by nasal canula. She continued to seize despite $6 \mathrm{mg}$ intravenous lorazepam and an intravenous fosphenytoin load. She was intubated and transferred to the intensive care unit on the neurology service for management of status epilepticus. In the intensive care unit she was given intravenous fosphenytoin and propofol. A lumbar puncture was performed for suspected encephalitis. Cerebrospinal fluid analysis, including evaluation for cryptococcus and herpes simplex virus, revealed no abnormalities. An MRI of her brain showed no acute abnormalities, and a work-up for an infectious etiology of her illness was negative. The infectious disease consultant suspected that the patient's seizures were a reaction to the moxifloxacin. It was discontinued and within twenty-four hours her seizures stopped. An EEG was performed the day after her seizure activity and intubation. The EEG showed diffuse 2-6 Hertz slowing secondary to sedation. No seizure or epileptiform abnormalities were seen. Three days after her initial intubation, she was successfully weaned from the ventilator. Her mental status quickly returned to baseline. Treatment with azithromycin was initiated for her respiratory infection, and at the recommendation of the neurologists, she was maintained on anti-epileptic 
medication. Her hospital course was complicated by an upper extremity DVT during her stay in the intensive care unit. Ten days after her initial admission she was discharged to her home with an outpatient enoxaparin bridge to warfarin. She was instructed to follow-up with her primary care provider, neurology, and infectious disease as an outpatient.

\section{Discussion}

In the absence of infectious or structural etiologies of the seizures, they were likely referable to moxifloxacin hydrochloride administration. Moxifloxacin is a normally well-tolerated fluoroquinolone antibiotic used to treat community-acquired pneumonia, sinusitis, and other bacterial infections. It does not require dose-modification for renal or hepatic impairment. Some common side-effects of moxifloxacin include upset stomach, vomiting, diarrhea, dizziness, and headache. The product information supplied with Avelox (brand name for moxifloxacin) notes that seizures are a rare complication of quinolone use, and have been reported with Avelox. It reports to "use moxifloxacin with caution in patients with known or suspected CNS disorders (for example, severe cerebral arteriosclerosis, epilepsy) or in the presence of other risk factors that may predispose to seizures or lower the seizure threshold."
Moxifloxacin has previously been reported in the literature as being associated with both seizures and status epilepticus. One case report described an elderly patient with a past history of idiopathic seizures who developed status epilepticus four days after initiating moxifloxacin treatment for a respiratory infection. His seizures stopped with discontinuation of the drug, but unfortunately he subsequently died of multiorgan failure associated with his illness. ${ }^{2}$ Additionally, an editorial noted that the WHO Adverse Reactions database contained 53 reports of convulsions associated with moxifloxacin. ${ }^{2}$

This report provides further evidence that moxifloxacin should be avoided, if possible, in patients with a history of seizure disorders. Furthermore, moxifloxacin should be considered as the possible cause of new onset seizures in a patient who has initiated treatment with this drug.

\section{References}

1. Product Information: AVELOX(R) oral tablets, IV injection, moxifloxacin hcl oral tablets, IV injection. Schering-Plough, Keniloworth, NJ, 2005.

2. Moxifloxacin: First report of status epilepticus in an elderly patient: case report. Reactions Weekly 2004; 995:11.
Ancient Rooftops, Seoul, Korea

Photo courtesy of Eric Choi, $M D$

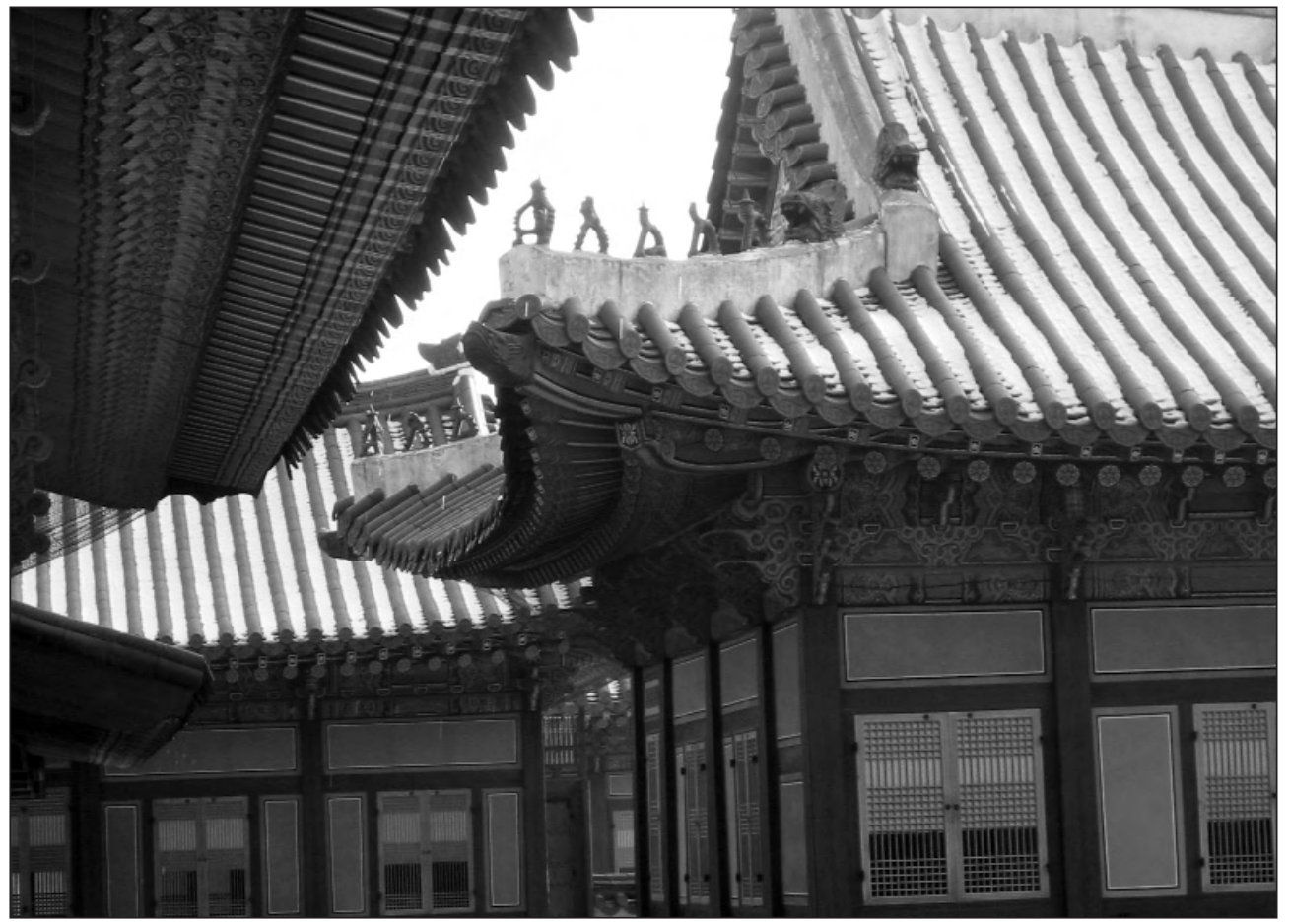

